\title{
Runtime Reconfiguration of FPGA for Biomedical Applications
}

\author{
Dipti Anil Puranik and Chetan Chugh \\ School of Electronics and Electrical Engineering, Lovely Professional University, Jalandhar - Delhi G.T. Road, \\ Phagwara - 144411, Punjab, India; difalguni@gmail.com, chetan.19004@lpu.co.in
}

\begin{abstract}
This paper describes the usage of Field Programmable Gate Array (FPGA) to explore reconfiguration to help out in the field of Biomedical Applications. The FPGA is reconfigured at runtime to analyze parameters like temperature, heart rate, blood pressure of patients and identify the conditions as normal, critical or emergency. According to the applications implemented there are two ways used to approach the Dynamic Partial Reconfiguration (DPR) practically. First is to configure the FPGA before reconfiguration and this part is known as static or fixed programming. Next part we reconfigure the FPGA again and run the program this is known as the partial reconfigurable part of the system. Basically initially two nodes are taken a temperature sensor along with the smoke sensor, after reconfiguration two more nodes are incorporated photo detector and Infrared Radiation (IR) sensor. Runtime reconfiguration is done to monitor parameters like blood pressure, heart rate, oxygen saturation etc. The runtime reconfiguration is carried out where we allow the user to enter the patient number and FPGA displays the condition of that particular patient at runtime. Hence a particular patients output can be observed. For which we can have $\mathrm{n}$ input patients and we can get the monitored continuous status of the nth patient. Such type of the program can be useful to send data wireless to the doctor and patient can be checked for the particular parameter. The outcomes involve displaying normal condition at temperature $98^{\circ} \mathrm{F} \&$ respiration rate of 17 inhalations per minute, displaying critical condition at temperature $102^{\circ} \mathrm{F} \&$ respiration rate of 30 inhalations per minute, displaying emergency condition at temperature $96^{\circ} \mathrm{F} \&$ respiration rate 127 inhalations per minute, emergency condition for the heart rate and the oxygen saturation levels of the patient while the temperature module keeps on function but not displayed. Also displayed is DPR for heart rate $70 \mathrm{bpm}$ and oxygen saturation of $95 \%$ indicates the normal condition, critical condition in DPR for heart rate $35 \mathrm{bpm}$ and oxygen saturation at $85 \%$, emergency condition in DPR for heart rate 165bpm and oxygen saturation at $60 \%$. Normal condition in DPR for bpl $85 \mathrm{mmHg}$ and bph $120 \mathrm{mmHg}$. The blood pressure of patient is monitored as normal condition in DPR for bpl $88 \mathrm{~mm} \mathrm{Hg}$ and bph $108 \mathrm{~mm} \mathrm{Hg}$. Normal condition in DPR18 for bpl $94 \mathrm{mmHg}$ and bph $150 \mathrm{mmHg}$. The above work can be successfully incorporated for patient health monitoring and can be further improved by sending wirelessly the data to a central hub if the patient's location is at some remote place.
\end{abstract}

Keywords: Application Specific Integrated Circuits (ASIC), Dynamic Partial Reconfiguration (DPR), ElectroCardioGraphy (ECG), Field Programmable Gate Array (FPGA), Head Of Bead (HOB), Hardware Descriptive Language (HDL), Internet Content Adaptation Protocol (ICAP), Integrated Synthesis Environment (ISE), Joint Test Action Group (JTAG), Joint Tactical Radio System (JTRS), Look Up Table (LUT), Partial Reconfiguration Modules (PRM), Patient Monitoring System (PMS), Wireless Sensor Network (WSN)

\section{Introduction}

The partial dynamic reconfiguration represents a novel exploration of flexibility of runtime reconfiguration of the WSN. In any large system vast amount of data is collected through multiple nodes in the system observing a particular event occurrence. Hence the interfacing and processing of each and every node becomes entirely different from one sensor point to another. Another major problem occurrence for such nodes is the rigid programmed microcontroller's. In case of microcontroller we observe two main drawbacks according to the current scenario of incorporating multiple nodes. First being the processing and application needs of the wireless network

\footnotetext{
*Author for correspondence
} 
increases rapidly which ultimately leads to the increase in the time - to - market demand. Also the microcontrollers are observed to avail limited sampling of input and output. With increase in number of nodes incorporation of new nodes become difficult. The program pattern once fed into the microcontroller cannot be altered from time to time. In case of any changes the entire program has to be restructured. To remove the above limitations and constrains arouse the need of hardware reconfiguration.

\subsection{Need of FPGA over Microcontroller}

FPGA's allow the abstraction of gate arrays where the freedom is to the developer to manipulate flip flops, inbuilt storage and logic gates. Runtime partial reconfiguration is also applicable in case of video systems. Major reasons to move to FPGA include the following factors improve implementation time, increase in confidentiality, removal of certain embedded constraints - restricted power consumed, thermal restrictions, dependability and particular event disturbance security. Along with these there is increase in control performance; execution time is reduced dynamically reconfiguring it.

Since 80's we observed the rapid growth of FPGA with several applications in variety of industries. A cutting edge has to be created by FPGA manufactures by updating their FPGAs in terms of technology. Currently a new model is seen developing in FPGA manufacturing so called DPR. Existing FPGAs are now been marketing which helps this feature available for the user. This new phase of FPGA used today has an advantage of more flexibility.

\subsection{DPR}

DPR provides a medium to change the logic implemented in FPGA when the device is kept working. DPR is only supported by few FPGAs for example in Xilinx Spartan 3, Virtex II, Virtex II Pro, Artix 7and Virtex 4.

In this report we shall be discussing more in detail about DPR and its terminology. There are many wellknown advantages of the DPR in spite of its difficult implementation. We shall study about the main parts utilized in its design flow.

\subsection{Dynamic Part}

Dynamic parts aren't required to be active during the whole lifetime of application hence are termed to be independent parts of input design. Inside target device modules share common areas (slots); as it is assumed that the modules do not require running parallel with other modules in the program at run time.

System scheduler decides upon which modules are to be programmed and not programmed inside the target device. Generally modules occupy memory outside FPGA as a bit stream. Changes may exist that the modules are stored inside the memory available in the FPGA.

\subsection{Static Part}

Parts that stay active throughout the runtime of the application are generally the static part for an input design. Always kept inside the static area of target and operating all the time. Apart from its own functioning it needs to establish a platform to load and unload additional dynamic modules in the design such as scheduling, managing data and interconnects between them.

It is necessary that this module must manage configuration controller and logical build up to perform interface and data management. Entire standard inputs and outputs of the system are done by the static modules communicating to the dynamic modules via fixed interfacing. The configuration of modules in memory and the instruction set for controlling and managing data is generated as a bit stream, which is transferred to the target via JTAG, USB, serial, or ICAP configuration interface.

Partial reconfiguration of FPGA is possible by using partial bit stream. The structure of device is modified using partial bit streams while the remaining of the program continues the run the similar way as before programmed.

\subsection{Applications of Reconfiguration}

Partial reconfiguration is a boon to enable changing of static modules of device at runtime and allowing the application to be created user specific and flexible to the environment. Partial reconfiguration is very much useful in building of memory architecture for reuse of space efficiently and so that the system works faster and better, it is also helpful in communication sector for end to end addition of encryption while transmitting and receiving of data, building of emulators, deployment of temperature sensors in forest fire areas, wireless sensor node communication among the various nodes at specific intervals, data acquisition in case of huge data handling processes and to aggregate the data in less memory space such that no waste of memory allocation exists and another field involving biomedical applications to help 
monitor the physical conditions of the patients. In this thesis we are primarily focusing on enhancement of the patient monitoring service. We can take the example of the biomedical application in wireless network using the smart suit build to check condition of the patient at runtime. The smart suit is utilized only for a particular cause, which handles the monitoring of physically challenged patients, with the entire physical data is gathered and sent to a hub. But such smart suit can also be put in practice of the monitoring of situation of the patient for emergency purposes?. To help check the condition of the patient for temperature sensing heart rate, respiratory rate of the body, arterial pressure and blood saturation rate in order to help diagnose the and bring in effectiveness of the electronic devices in day to day life of the patient. The most important thing currently is deployment of the patient monitoring systems at cost effective and at efficient rate. However there are still challenges to overcome. Since these systems have to be operated with human body there exist the material constraints. Moreover there must be robustness and high fault tolerance rate in such systems. Recently electronic devices at low cost sensors have made way in medical field to be used in daily life. The olden blood pressure pumping devices are being replaced by the new electronic devices no need of manual pumping mechanism, once the device is adjusted and switched on it operates the pressure check function on its own.

Today's technology E- textiles have been flourishing where in electronics and textiles have collaborated together to the build a mass production of garments and help manufacture rate of textile industry to be increased. It is the collective procedure of gathering minute data, observing statistical movements and processing it further for remote monitoring.

Similarly there are abundant portable electronic units available today which meets the diagnostic requirement of the patient. When such units are incorporated together it leads to the development of a smart suit embedded suitable for the medical applications.

It is simple to imagine an athlete wired whose physical body parameters are analyzed to check the critical condition of him and help assist emergency medical aid ${ }^{5}$. Several flexible solutions of health monitoring are being implemented in WSN. Some systems are based on improving the network reconfiguration. Others are based upon mobile virtual machines to reprogram the system. Such networks help remove the gap between the low level end and high level end abstraction helping system to help get used to the changes in the environmental situations.

In this work the model is expanded to provide indications of health check emergency care of the patient. The dynamic reconfiguration is related to the actual patient condition.

The physiological data is collected by the smart suit which is embedded with units sensing the wired raw data and route it to the processor for further parameter check. The suit can monitor the cardiac rate, respiratory rate, arterial pressure rate, and body temperature and blood saturation rate. Figure 1 shows the smart suit unit for physiological data acquisition.



Figure 1. Smart suit unit for physiological data acquisition.

\subsection{Physiological Measuring Parameters}

The system is based upon the checking the data obtained by the sensors and availing necessary information for taking immediate precautions within time. For measuring cardiac and respiratory rates sensors are positioned in shirts around patient's chest and abdomen area. A network of CMOS temperature sensor tells about body temperature. Different sensors are embedded and prove a good example of combination of textile and electronics together. This system can also further expand in wireless/medium. The following parameters are briefly explained for the emergency care required to be taken. The physiological data is recorded by the system, such as cardio rates, temperature, blood pressure, respirations and blood saturation. Normally in this function, the sampling of information is done at a low rate and sent to the hub in order to process, analyze and visualize the data. The FPGA can choose only particular set of signals to analyze it in detail, in order to reduce the required data rate so that the network can handle it. It is also important the system has to continue to operate even after reconfiguration also the time required for reconfiguration must not interfere with 
the data to process and data acquisition. Thus dynamic reconfiguration helps for the runtime reconfiguration of the condition parameters. The following explains the parameters utilized in the processing.

\subsubsection{ECG / Heart Rate}

The electrocardiograph is an instrument used to measure the heart activity. The time variant voltage produced by the instrument, which is being recorded is called electrocardiogram.

The P, Q, R, S, T waves reflect the basic waveforms of electrocardiogram i.e. rhythmic electrical depolarization and repolarisation of the myocardium related with the contraction and expansion of the atria and ventricles. The electrocardiogram is used in diagnosing various diseases and conditions linked with the heart. We take in signal from such a unit indicating the heart beats per minute rate to find out our critical condition parameters. Certain important ECG parameters are discussed here. The shape and time of each feature of the ECG is significant. The cardiologist looks critically at the various time intervals, polarities and amplitude to arrive at his diagnosis.

Some normal values of amplitude and time interval:

\subsubsection{Amplitude}

Table 1 shows the amplitude for each type of wave.

Table 1. Amplitude for each type of wave

\begin{tabular}{ll}
\hline Sr No. & Amplitude \\
\hline (i) & P wave $0.25 \mathrm{mV}$ \\
(ii) & R wave $1.6 \mathrm{mV}$ \\
(iii) & Q wave $25 \%$ of $\mathrm{R}$ wave \\
(iv) & T wave 0.1 to $0.5 \mathrm{mV}$ \\
\hline
\end{tabular}

\subsubsection{Time Interval}

Table 2 shows the time interval for each type of wave.

Table 2. Time interval for each type of wave

\begin{tabular}{|c|c|}
\hline Sr No. & Duration \\
\hline (i) & $\begin{array}{l}\text { P-R Interval } 0.12 \text { to } \\
0.20 \mathrm{sec}\end{array}$ \\
\hline (ii) & $\begin{array}{l}\text { Q-T interval } 0.35 \text { to } \\
0.44 \mathrm{sec}\end{array}$ \\
\hline (iii) & $\begin{array}{l}\text { S-T segment } 0.05 \text { to } \\
0.15 \mathrm{sec}\end{array}$ \\
\hline (iv) & $P$ wave interval $0.11 \mathrm{sec}$ \\
\hline (v) & QRS interval $0.09 \mathrm{sec}$ \\
\hline
\end{tabular}

For the patient's diagnosis following steps are to be followed by cardiologist:

- First he looks at his heart rate. The normal values lies in range of 60 to 100 beats per min. A slow rate than this is called bradycardia less than 40 beats per minute. A higher rate than this rate is called tachycardia greater than 160 beats per minute.

- Then he will see the cycles. If they are not evenly placed, arrhythmia is indicated.

- If the P-R intervals is greater than $0.2 \mathrm{sec}$, it suggest blockage of the AV node.

- If one or more basic feature of the ECG is missing, a heart block of some sort might be indicated.

In pathological condition several changes may occurs in ECG:

- Altered path of excitation in the heart.

- Changed origin of waves

- Altered relationships of features

- Changed magnitude of one or more features

- Differing duration of waves or intervals.

With the instrument electrocardiograph diagnosis of cardiac disorder can be done easily. Apart from the heart beat rate the graphical plotting of the ECG also is very essential to diagnose a case. The continuous ECG monitoring is standard for patients who are at high risk for dysrhythmias. Figure 2 shows normal and Abnormal ECG.

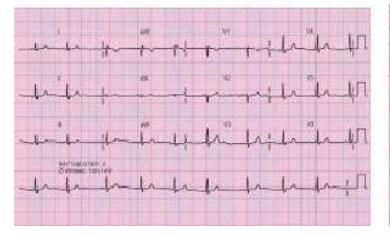

Figure 2. (a) Normal ECG.

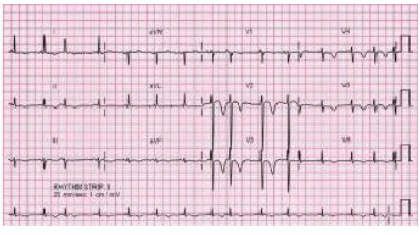

(b) Abnormal ECG.

\subsubsection{Pulse oximetry}

It is a noninvasive method of constantly monitoring the oxygen saturation of hemoglobin ( $\mathrm{SpO} 2$ or $\mathrm{SaO} 2)$. Pulse oximeter does not substitute arterial blood gas measurement; it is an effective tool to monitor for slight or sudden changes in oxygen saturation. Figure 3 shows Oximeter sensor device. 


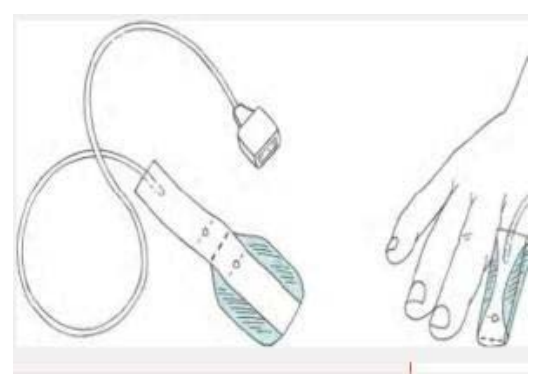

Figure 3. Oximeter sensor device.

It is utilized for all settings where oxygen saturation monitoring is required, such as the home, clinics, ambulatory surgical settings, and hospitals.

A probe or sensor is connected to the fingertip, forehead, earlobe or bridge of the nose. The sensor detects changes in oxygen saturation levels by observing light signals generated by the oximeter and reflected by the blood pulsing through the tissue at the probe.

Table 3 shows Spo 2 levels and its interventions.

Table 3. Spo2 levels and its interventions

\begin{tabular}{|c|c|}
\hline Finding & Interventions \\
\hline SpO2>95\% & $\begin{array}{l}\text { Normal, no intervention needed. } \\
\text { Continuous routine monitoring of patient. }\end{array}$ \\
\hline SpO2 91-94\% & $\begin{array}{l}\text { Acceptable, check probe placement and } \\
\text { adjust if needed. Continue to monitor } \\
\text { patient. }\end{array}$ \\
\hline $\mathrm{SpO} 285-90 \%$ & $\begin{array}{l}\text { Raise head of bed (HOB) and allow patient } \\
\text { to breathe deeply. Assess airway and } \\
\text { encourage coughing. Suction airway if } \\
\text { needed. Administer oxygen and titrate to } \\
\text { SpO2 }>90 \% \text {. Notify physician and respira- } \\
\text { tory therapist (RT) if SpO2 fails to improve } \\
\text { after few minutes. }\end{array}$ \\
\hline $\mathrm{SpO} 2<85 \%$ & $\begin{array}{l}\text { Administer } 100 \% \text { oxygen, position patient } \\
\text { to facilitate breathing, suction airway } \\
\text { if needed, and notify physician and RT } \\
\text { immediately. }\end{array}$ \\
\hline
\end{tabular}

\subsubsection{Blood Pressure}

To determine blood pressure of an individual is one of the most important and useful clinical measurement and hence the most frequently measured pressure. Blood pressure means the pressure exerted by the blood against the vessel walls. One should become familiar with the following pressures:-

\subsubsection{Systolic blood pressure}

The highest pressure in the artery, created in the heart's contraction (systolic) phase. The normal value for a 20 -year-old man is $120 \mathrm{~mm} \mathrm{Hg}$.

\subsubsection{Diastolic blood pressure}

The lowest pressure in the artery, created in the heart's relaxation (diastolic) phase. The normal value for a 20 -year-old man is $80 \mathrm{~mm} \mathrm{Hg}$.

\subsubsection{Pulse pressure}

The difference between the systolic and diastolic pressures. The normal value is $40 \mathrm{~mm} \mathrm{Hg}$.

\subsubsection{Mean blood pressure}

Diastolic pressure plus one third of the pulse pressure. This is the average effective pressure forcing blood through the circulatory system. The normal value is 96 to $100 \mathrm{~mm} \mathrm{Hg}$.

Blood pressure can be measured by several techniques. They are categorized into two main methods:-

i. Direct method:

The direct method requires direct insertion of a tube or catheter into a blood vessel. The catheter is connected to a blood pressure transducer, which generates an electrical signal.

ii. Indirect method:

In this method, we measure the blood pressure using two different methods:

- The first method use sense of touch: called the palpatory method.

- The second method use the sense of hearing: called the auscultatory method.

In both indirect methods, using an instrument called the sphygmomanometer pressure is applied to the artery. Sphygmomanometer is an instrument that measures pressure. Each sphygmomanometer consists of a cuff which is connected by lengths of tubing to an inflating bulb with a needle valve and to a mercury manometer.

\subsubsection{Body Temperature}

The human body temperature is also basic important parameter for indication of levels of rise in the body temperature. Usually our body temperature is normal 
at about 98 degree Fahrenheit (F) (i.e. between 36.5 and 37.4 degree Celsius). For above 98 degree $\mathrm{F}$ we take in precautions as this phase is termed as hyperthermia. And for levels below 95 degree $\mathrm{F}$ the condition is termed as hypothermia. Any value greater or less than the normal value requires the urgent attention for the health condition.

\subsubsection{Respiratory Rate}

Inhalation and exhalation both together creates respiration. Human respiration rate is measured during the resting phase of a person and number of breathes are done by counting the number of breaths for one minute which is done by counting number of times the chest rises. Observation of patients currently is done by an optical breath rate sensor. Respiration rates also may increase by fever, ill health, or other ill conditions. When observing respiration, it is important to also consider whether a person has any other breathing difficulty.

Varied methods including impedance pneumography and capnography are commonly used technique in measuring respiration rate. In addition new techniques to automatically observe respiratory rate using mobile sensors are in development.

Normal range: For a healthy adult at rest respiratory rate is $12-20$ breaths per minute. Average resting respiratory rates by age are shown in Table 4 .

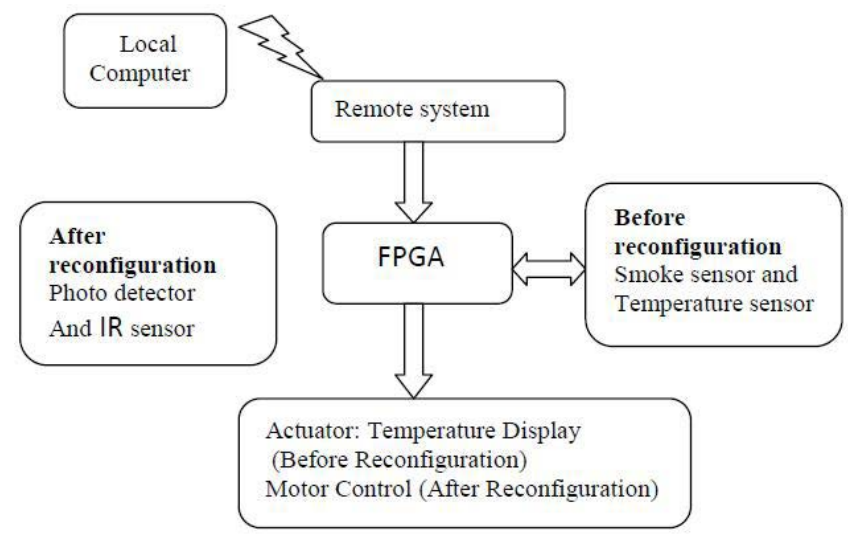

Figure 4. Block diagram of methodology used.

Evaluation of respiratory rate in babies less than 6 months for the differentiating severity of illness found it not to be very helpful. Also factors such as cry, sleep, agitation and age cause significant influence on the respiratory rate. Because of these factors and similar studies the indications of respiratory rate for serious illness is limited.

It is most important to check the physical condition of intensely-ill patients. It is measured continuously to provide detection of alterations in physical health.

\section{Scope of the Study}

\subsection{Applications}

Varied and large in number industries have accepted the new concept of partial reconfiguration and even put into implementation. Major Fields such as aerospace and defence industries clearly have taken advantage of its capacity to reconfigure. Example such as the Joint Tactical Radio System (JTRS) Program has benefited a lot by partial reconfiguration.

\subsection{Increased System Performance}

Even though only a part of system is redesigned the remaining functions continue to operate normally. Such systems provide high rate of performance as the failure of a particular module does not affect the other module's functionality - no downtime. It enables the user to handle multiple applications on FPGA.

\subsection{The Ability to Change Hardware}

Xilinx FPGAs are easy to update at any time, locally or remotely. Partial reconfiguration makes the user available to support easily, guarantee service and upgrade hardware in the field area.

\subsection{Hardware Sharing}

By allowing the FPGA to process multiple applications at runtime we can observe the hardware is shared among them. Advantages of this are reduced machine count, reduced power consumed, smaller board size, and overall lower prices.

\subsection{Shorter Reconfiguration Times 8}

Configuration time is directly proportional to the size of the configuration bitstream. Partial reconfiguration benefits us to perform small changes without having to change the entire system. Only by changing only parts 
of bitstream - as observed opposite to reconfiguring the entire system - the entire reconfiguration time is much less'.

\section{Research Methodology}

In order to implement a partial reconfiguration design successfully, we follow certain design methodologies which are described below:

One among the common method used in Plan Ahead tool is -

- Insert bus macros between modules that are required to be interchanged out (partial reconfiguration modules, or PRMs) and the remaining design is static logic. They become the channel/ ports by which modules communicate and transfer data.

- Synthesis guidelines to be followed to generate a netlist partially reconfigurable.

- Carry out floor planning of the PRMs and collect all static modules together.

- Provide bus macros.

- Follow PR-specific design rules.

- Run the partial reconfiguration implementation flow.

\subsection{Bus Macros}

Bus macros are useful for predetermined data paths for signals to flow between a dynamic module and any other module. The HDL code should keep a track that any reconfigurable module signal that communicates with other module happens only by first transiting signal through a bus macro.

The chosen device must be compatible to the instantiated version. Every bus macro gives a 4 bit of inter-module connection. The number of bus macros must be instantiated to equal the number of bits travelling the borders of the dynamic modules.

"An example, if reconfigurable module A communicates via 32 bits to module B, then eight (32/4) bus macros will be required to be instantiated to form the data paths between modules A and B." [6]. Dataflow in bus macro is forever unidirectional. So Bus macros are setup for flow of data in unidirectional rigid path. Xilinx provides these Bus Macros. It is also possible to design our own bus micros.

\subsection{Configuration Controller}

Configuration controllers/microcontrollers are a part of the static module. They take care to load and unload the dynamic programs. Configuration controller makes the design self-sufficient. Which means reconfiguration does not need any human interference. All we need to tell the microcontroller when it has to configure and which module. This is possible by ' $C$ ' program or assembly program language depending on the user flexibility.

With help of Xilinx EDK tool these controllers can be configured as a configuration controller. Certain constrains are applied during configuration of the FPGA.

\subsection{Study of Proposed Work}

According to the applications implemented there are two ways to approach the DPR practically. First is to configure the FPGA before reconfiguration and this part is known as static or fixed programming. Next part we reconfigure the FPGA again and run the program this is known as the partial reconfigurable part of the system . Basically initially two nodes are taken a temperature sensor along with the smoke sensor, after reconfiguration two more nodes are incorporated photo detector and IR sensor. These pair of nodes before and after reconfiguration is utilized to run a stepper motor actuator. The FPGA is connected to the software simulator using JTAG cable. In Virtex 4 we shall make use of USB cable.

The following diagram explains the methodology used in order to reconfigure the FPGA where we have 2 modules before and after reconfiguration 1 each consisting of sensors interfaced with FPGA. Figure 4 shows the block diagram of methodology used.

Table 4. Agewise respiration rate

\begin{tabular}{ll}
\hline Age & $\begin{array}{l}\text { Respiration } \\
\text { rate(inhalalations per } \\
\text { minute) }\end{array}$ \\
\hline Birth to 6 weeks & $30-60$ \\
6 months & $25-40$ \\
3 years & $20-30$ \\
6 years & $18-25$ \\
10 years & $17-23$ \\
Adults & $12-18$ \\
$>=65$ years & $12-28$ \\
$>=80$ years & $10-30$ \\
\hline
\end{tabular}


The program is first run by using the temperature sensor's data as input and if the temperature exceeds 50 degrees then the smoke sensor is made active and the temperature is displayed on 7 segment display as $\mathrm{L}$ and $\mathrm{H}$. Next the reconfiguration is done where motor and IR sensor are connected. When the IR sensor's signal is detected then the motor stops running. Next the photo detector is interfaced dynamically and the signal from IR sensor and photo detector now control the motor.

Results drawn from the experiment done are compared in the graphs. After the simulation it was observe that 2 bit files are generated one for fixed module and other for reconfiguration module. Their file size is $1117 \mathrm{~kb}$ and $84 \mathrm{~kb}$ respectively. The bandwidth maximum utilised is $66 \mathrm{Mbps}$. Time taken to send the files are $0.002 \mathrm{sec}$ and $0.00015 \mathrm{sec}$ respectively. Overall time taken to send the complete configuration is $49 \mathrm{~s}$ and $7 \mathrm{~s}$ in fixed and partial respectively according to the base paper implementations.

Hence we observe that DRC helps to reduce memory utilized, Time taken for delivery of bit files and Bandwidth utilized is small during the runtime. Additional to all these features the Virtex - 4 based FPGA is found to be the least power consuming device. Figure 5 shows flowchart before reconfiguration of PMS. Figure 6 shows flowchart before reconfiguration of PMS.



Figure 5. Flowchart before reconfiguration of PMS.

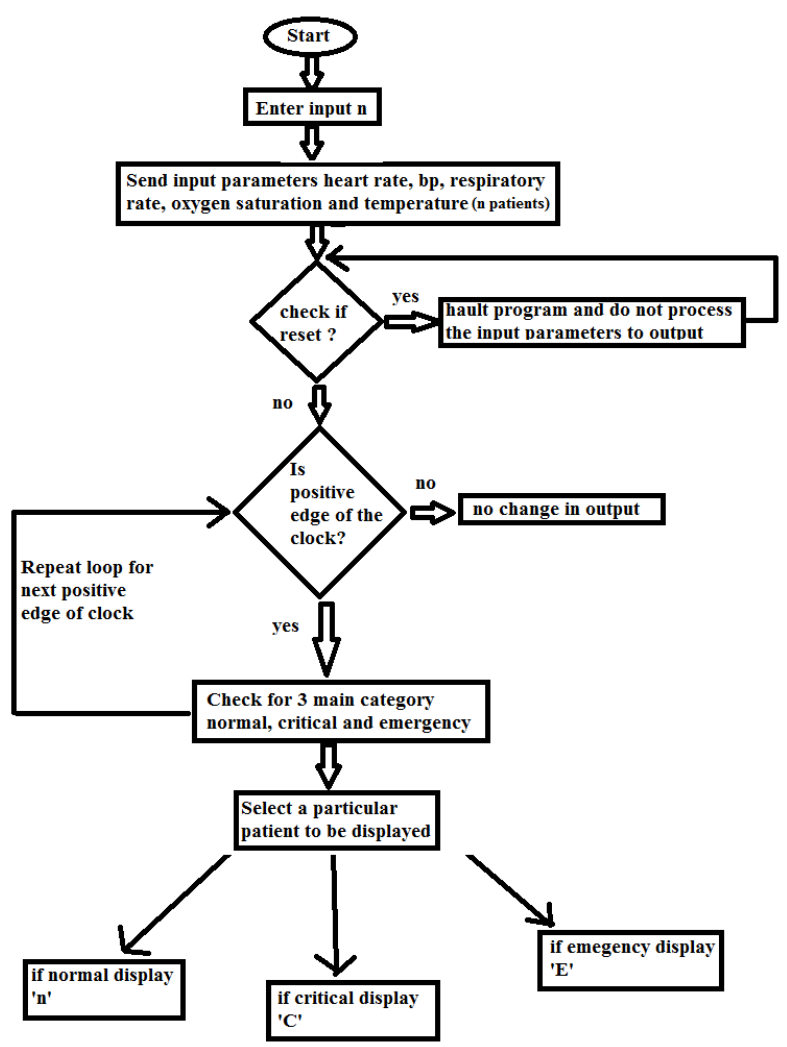

Figure 6. Flowchart for the PMS after reconfiguration.

\section{Implementation}

Methodology used follows the following steps were carried out in order to study the dynamic reconfiguration of the FPGA board. The static and partial blocks where defined as will be discussed stepwise below.

- Temperature sensor and led is configured before reconfiguration. Temperature is sensed given as input parameter.

- Motor movement is configured after reconfiguration.

- FPGA is connected to the laptop using either JTAG or an USB cable.

- Temperature sensor and led are programmed whose output now controls the output of stepper motor. If temperature value $>50 \mathrm{deg}$ then motor stops, else if value of sensor goes low motor rotates in anticlockwise direction.

- The fixed and partial module work together after reconfiguration ${ }^{6}$. 
Figure 7 below shows the bar graph of comparison of fixed and reconfiguration data in terms of memory and time.

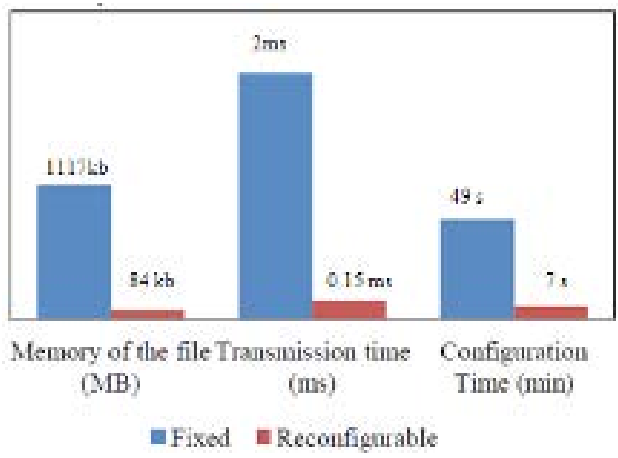

Figure 7. Comparison table for fixed and reconfigurable modules.

Figure 8 contains the seven segment display when (a) temp $>50$ deg and fire smoke alarm is generated, (b) temp $>50$ deg but no alarm generated and (c) temp $<50$ deg. before reconfiguration.



(a)



(b)



(c)

Figure 8. Above shown figures contain the seven segment display when (a) temp $>50 \mathrm{deg}$ and fire smoke alarm is generated, (b) temp $>50$ deg but no alarm generated and (c) temp $<50 \mathrm{deg}$. before reconfiguration.

The simulations below are for the motor rotation after reconfiguration. Here the speed of motor isn't paid much attention as rather only the output check of reconfiguration during runtime is studied by this experiment. Table 5 shows the Motor rotation after reconfiguration. Figure 9 shows the initial step of the module configuration of temperature enabling. Figure 10 shows the Normal configuration of temperature sensor and led. Figure 11 shows simulation after reconfiguration.

Table 5. Motor rotation after reconfiguration

\begin{tabular}{lcccc}
\hline CLk & $\begin{array}{c}\text { Temp } \\
\text { enable }\end{array}$ & $\begin{array}{c}\text { Motor } \\
\text { enable }\end{array}$ & $\begin{array}{c}\text { Motor } \\
\text { output 1 }\end{array}$ & $\begin{array}{c}\text { Motor } \\
\text { output2 }\end{array}$ \\
\hline 1 & 0 & 0 & 0 & 0 \\
1 & 1 & 0 & 0 & 0 \\
1 & 1 & 1 & 0 & 1 \\
1 & 1 & 0 & 0 & 1 \\
\hline
\end{tabular}

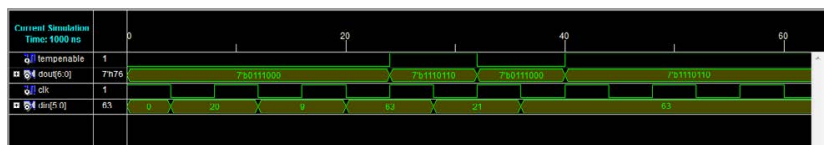

Figure 9. FInitial step of the module configuration of temperature enabling.

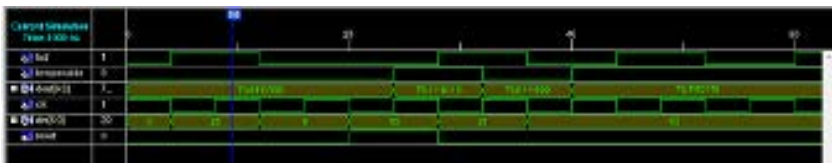

Figure 10. Normal configuration of temperature sensor and led. 


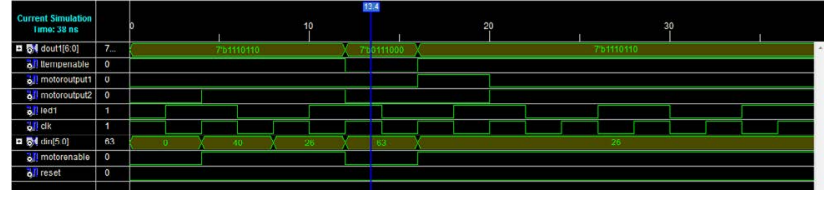

Figure 11. NSimulation after reconfiguration.

The FPGA can also be used in order to aggregate the data from multiple sensor nodes and then send the processed aggregated data further to microcontroller for various operations according to the application. Figure 12 shows the block diagram of data aggregation using FPGA.

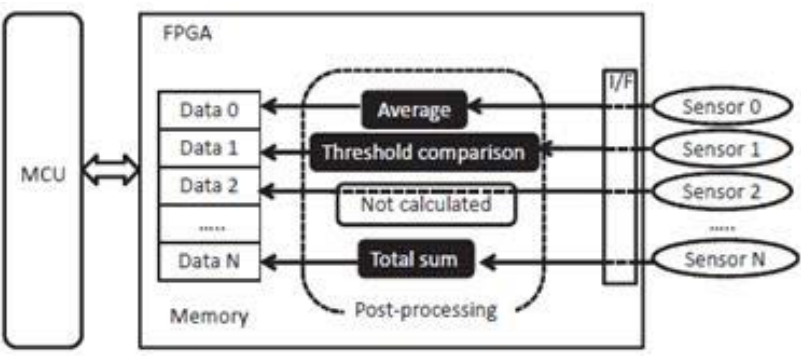

Figure 12. Block diagram of data aggregation using FPGA.

The above experimental work proposed is significant and outputs generated are also according to the expectations. Using the above concept the further building of application specific PMS (Patient Monitoring System) is been developed for which the concept being to generate the emergency care indications for the patient at runtime and reconfiguration any data for specific patient.

The methodology utilized is similar as above as the modules to be configured are subdivided into two main parts static and dynamic parts. The dynamic modules are into 5 sub categories depending upon the 5 input measured conditions of the patient. Each sub categorized module is necessary to be having flow of data from main static module using the bus macros. The data is collected and processed further accordingly by the health conditions $\mathrm{t}^{2}$. We have first the work done upon the patient monitoring of a single patient. Following are the output parameters for the health monitoring of a single patient where the outputs are displayed upon the seven segment display. The patient condition is checked for all the 3 conditions where the first cycle is for the normal condition for all the five parameters, the next cycle includes the critical region and the third cycle indicates the emergency levels. All the parameters can be wirelessly transmitted if the patient is at remote place $\left.\right|^{\text {月 }}$.

Table 6 shows the parameter categorized into normal, critical and emergency. Figure 13 shows five input parameters of the patient. Figure 14 shows five output display parameters in seven segment display.

Table 6. Parameter categorized into normal, critical and emergency

\begin{tabular}{|c|c|c|c|}
\hline Parameter & Normal & Critical & Emergency \\
\hline $\begin{array}{l}\text { Temperature (degree } \\
\text { Fahrenheit) }\end{array}$ & 98 & $>99$ & $<97$ \\
\hline $\begin{array}{l}\text { Heart rate (beats per } \\
\text { minute) }\end{array}$ & $60-100$ & $<40$ & $>160$ \\
\hline Oxygen saturation (\%) & $90-100$ & $85-90$ & $<85$ \\
\hline $\begin{array}{l}\text { Blood pressure } \\
(\mathrm{mmHg})\end{array}$ & $120 / 85$ & $\begin{array}{c}\mathrm{Bph}< \\
110 / 85\end{array}$ & $\begin{array}{c}\text { Bph }>140, \\
\text { Bpl }>90\end{array}$ \\
\hline \multirow{2}{*}{$\begin{array}{l}\text { Respiratory rate (inha- } \\
\text { lations per minute) }\end{array}$} & $16-18$ & $>25 \mathrm{Or}$ & $>45 \mathrm{Or}$ \\
\hline & & $<10$ & $<5$ \\
\hline
\end{tabular}
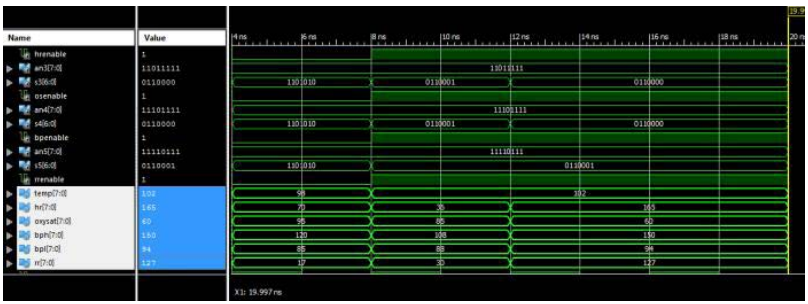

Figure 13. Five Input parameters of the patient.

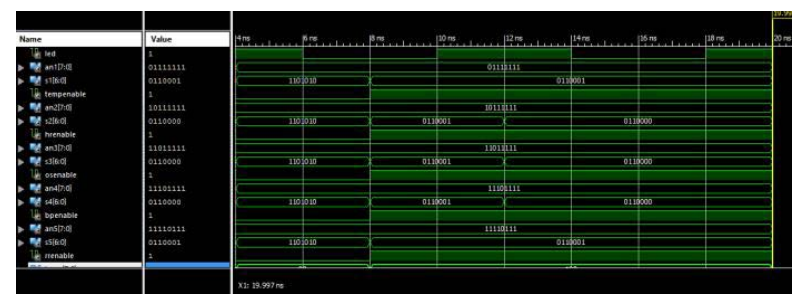

Figure 14. Five output display parameters on seven segment display.

The next part includes the reconfiguration of the data for several patients taken by the input parameter ' $n$ '. For $\mathrm{n}$ input patients the output is stored and processed accordingly in similar manner as done for the single patient. The reconfiguration provides the user to incorporate many patients as well as display the output of a particular patient when selected. Such is an advantage to change the processing in accordance with the changes in the environment. 
Below given is the pictorial representation of the RTL schematics for the partial as well as static modules in the dissertation II part. We can observe that the bus interfaces leave the main static module and reconfigurable modules are all in link to the top level module. Figure 15 shows RTL schematic of the temperature and motor movement dynamic reconfiguration. Figure 16 shows RTL schematic representation of the patient monitoring system.

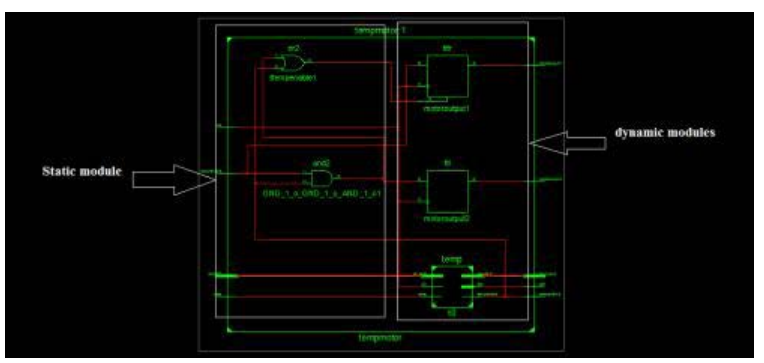

Figure 15. RTL schematic of the temperature and motor movement dynamic reconfiguration.

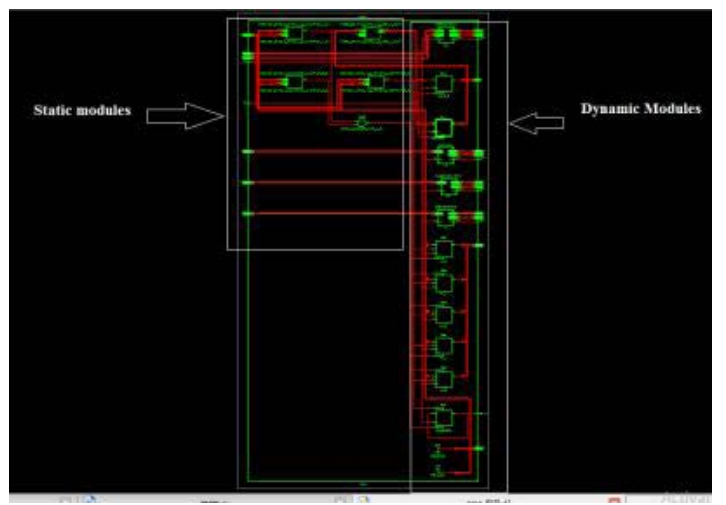

Figure 16. RTL schematic representation of the patient monitoring system.

\section{Results and Discussions}

Artix series FPGA is capable to the DPR but the existing reconfiguration conflict significantly restricts in terms of numbers of reconfigurable units the scale of the system. In this work we discussed about present dynamic reconfiguration techniques, considering medium such as Xilinx Virtex, Virtex-II,Virtex-II Pro FPGA,Artix-7 etc. Practical application result of the DPR is discussed where following represents the expected outcomes we achieved while performing reconfiguration.

Due to the resource constraints and the limitations on the FPGA board available the following possible outcomes are discussed herein. The FPGA comprises of only 16 input switches and 8 seven segment display on board. We can view the Nexys4 Artix - 7 Board in Figure 17.

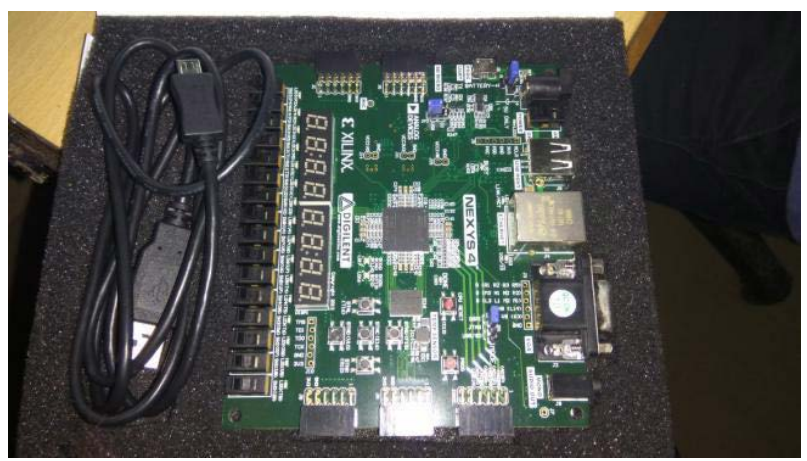

Figure 17. Nexys4 Artix 7 FPGA kit.

The board has an inbuilt internal clock pulse generator of $100 \mathrm{MHz}$ frequency and the period of $10 \mathrm{~ns}$ and $50 \%$ duty cycle waveform. The seven segment display having common anode configuration has an inverter pmos transistor input hence in order to activate the seven segment display we need to give the active low input at the anode. Whereas the cathode terminal of the diode are all given active high level inputs to illuminate the desired sign upon the display. Studying the lab manual configuration of board the connectivity of switches and micro switches or push buttons to the seven segment display can be achieved.

The expected outcomes for the work were limited to be shown together due the input constraints of the kit. Hence the output of the PMS has been achievable by using dynamic runtime configuration to display the emergency conditions of the patient. The main module comprises of the 3 functions continuous working led blinking indicator, the temperature sensing module and dynamic respiration rate modulef. The below output is the working of the above modules according to the emergency indication conditions with reference to the previous chapter table. Figure 18 displays normal condition at temperature $98{ }^{\circ} \mathrm{F} \&$ respiration rate of 17 inhalations per minute. Figure 19 displays critical condition at temperature $102^{\circ} \mathrm{F} \&$ respiration rate of 30 inhalations per minute. Figure 20 displays emergency condition at temperature $96^{\circ} \mathrm{F}$ \& respiration rate 127 inhalations per minute. After creating the main module we now carry out the dynamic runtime reconfiguration 1 and we can monitor and display emergency condition for the heart rate and the oxygen saturation levels of the patient while the 
temperature module keeps on function but not displayed. Figure 21 shows DPR for heart rate $70 \mathrm{bpm}$ and oxygen saturation of $95 \%$ indicates the normal condition. Figure 22 displays critical condition in DPR for heart rate 35 bpm and oxygen saturation at $85 \%$. Figure 23 displays emergency condition in DPR for heart rate $165 \mathrm{bpm}$ and oxygen saturation at $60 \%$.

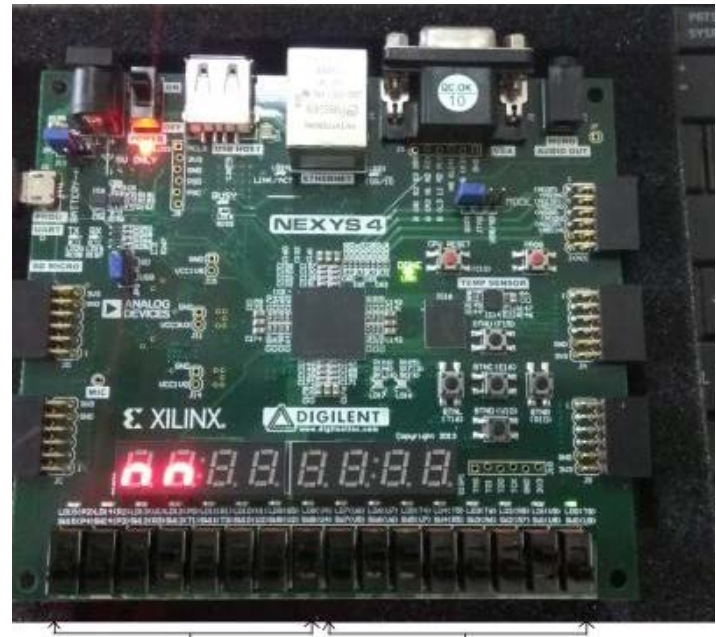

respiration rate $=17$

temp $=98$ degree $F$ (inhalations per minute)

Figure 18. Displaying normal condition at temperature 98 ${ }^{\circ} \mathrm{F} \&$ respiration rate of 17 inhalations per minute.



Figure 19. Displaying critical condition at temperature 102 ${ }^{\circ} \mathrm{F} \&$ respiration rate of 30 inhalations per minute.

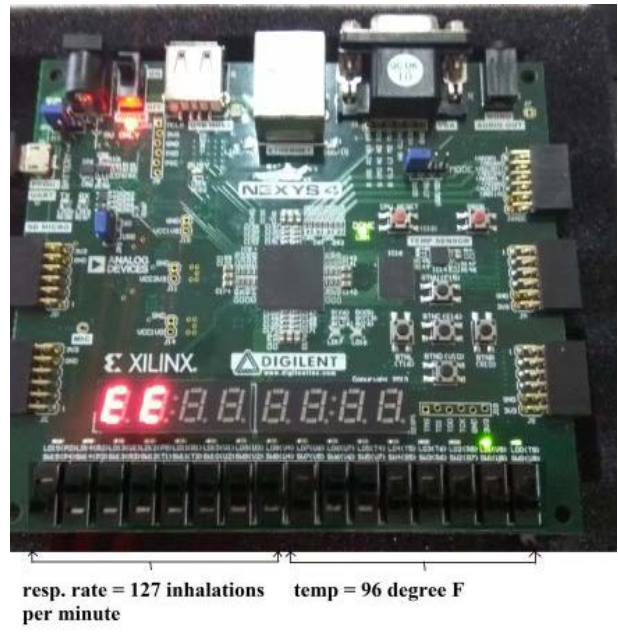

Figure 20. Displaying emergency condition at temperature $96^{\circ} \mathrm{F} \&$ respiration rate 127 inhalations per minute.

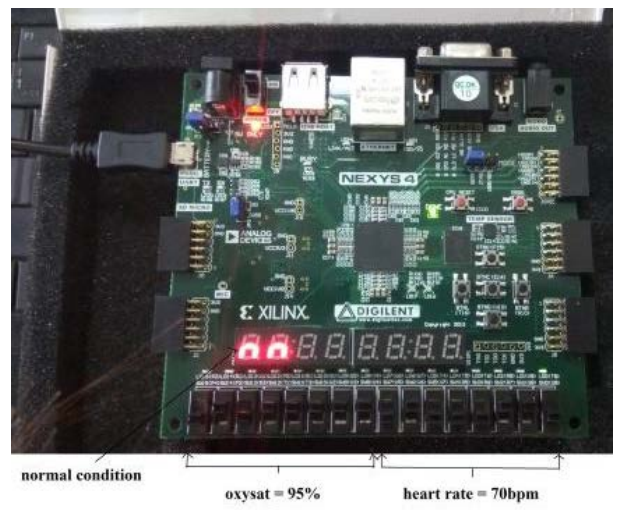

Figure 21. DPR for heart rate 70bpm and oxygen saturation of $95 \%$ indicates the normal condition.

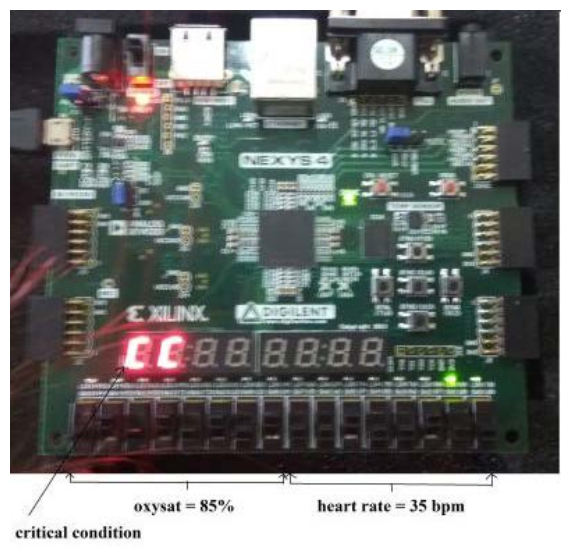

Figure 22. Displaying critical condition in DPR for heart rate $35 \mathrm{bpm}$ and oxygen saturation at $85 \%$. 




Figure 23. Displaying emergency condition in DPR for heart rate $165 \mathrm{bpm}$ and oxygen saturation at $60 \%$.

Further the runtime reconfiguration is done for the display of blood pressure levels 1 . There are two levels to check in case of arterial pressure the high and low blood pressure. When both the high and low levels of condition are satisfied then we can get an emergency condition output on the seven segment display. Let us see the 3 outputs of the blood pressure for all the conditions.

Figure 24 displays normal condition in DPR for bpl $85 \mathrm{mmHg}$ and bph $120 \mathrm{mmHg}$. Figure 25 displays normal condition in DPR for bpl $88 \mathrm{mmHg}$ and bph $108 \mathrm{mmHg}$. Figure 26 displays normal condition in DPR for bpl 94 $\mathrm{mmHg}$ and bph $150 \mathrm{mmHg}$.

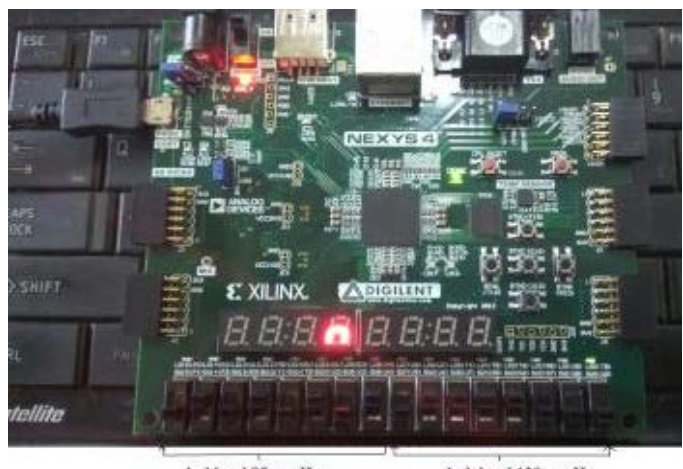

bpl level $85 \mathrm{mmHg}$

bph level $120 \mathrm{mmHg}$

Figure 24. Displaying normal condition in DPR for bpl 85 $\mathrm{mmHg}$ and bph $120 \mathrm{mmHg}$.

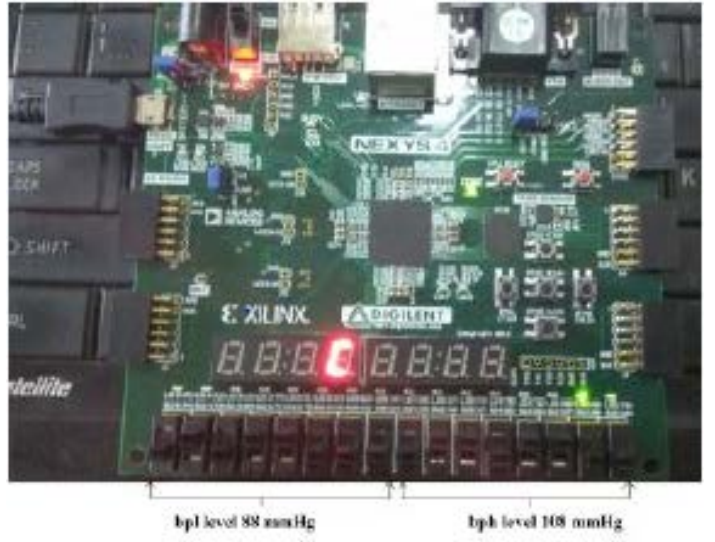

Figure 25. Displaying normal condition in DPR for bpl 88 $\mathrm{mmHg}$ and bph $108 \mathrm{mmHg}$.



Figure 26. Displaying normal condition in DPR for bpl 94 $\mathrm{mmHg}$ and bph $150 \mathrm{mmHg}$.

Lastly then we carry out the runtime reconfiguration where we allow the user to enter the patient number and FPGA displays the condition of that particular patient at runtime. Hence a particular patients output can be observed. For which we can have $\mathrm{n}$ input patients and we can get the monitored continuous status of the nth patient. Such type of the program can be useful to send

Data wireless to the doctor and patient can be checked for the particular parameter. Below are the results of the $5^{\text {th }}$ patient monitored at runtime for all the input parameters. Figure 27 shows fifth patient monitoring at normal condition for temperature. 
Figure 28 shows fifth patient monitoring at critical condition for temperature. Figure 29 shows fifth patient monitoring at emergency condition for temperature.

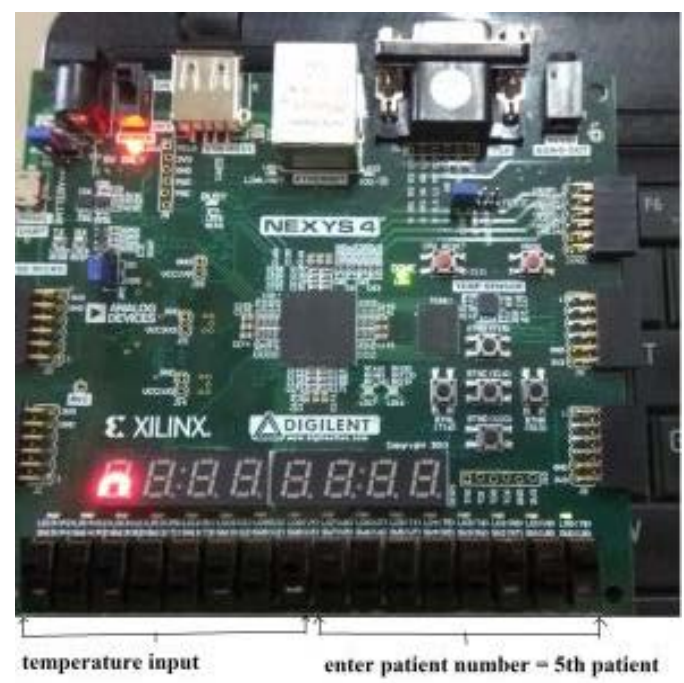

Figure 27. Fifth patient monitoring at normal condition for temperature.

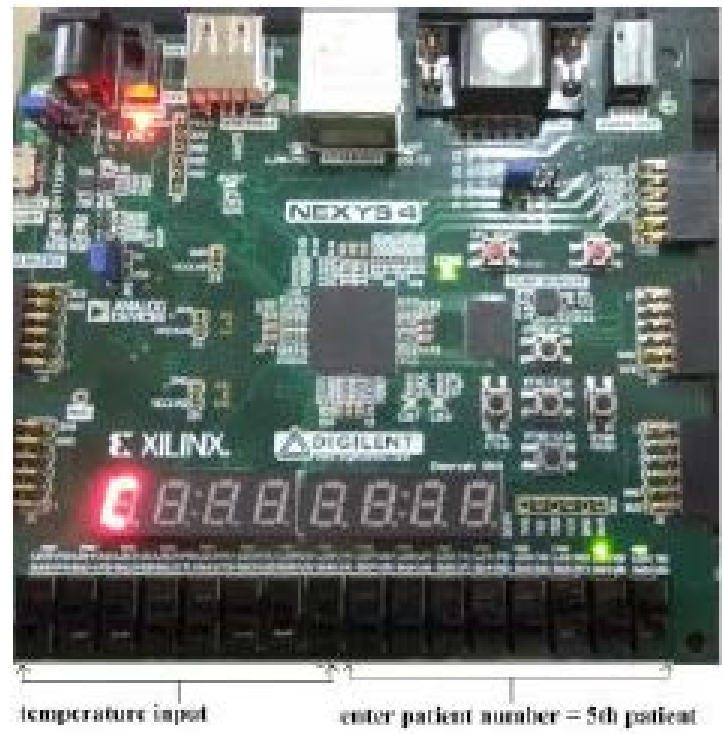

Figure 28. Fifth patient monitoring at critical condition for temperature.

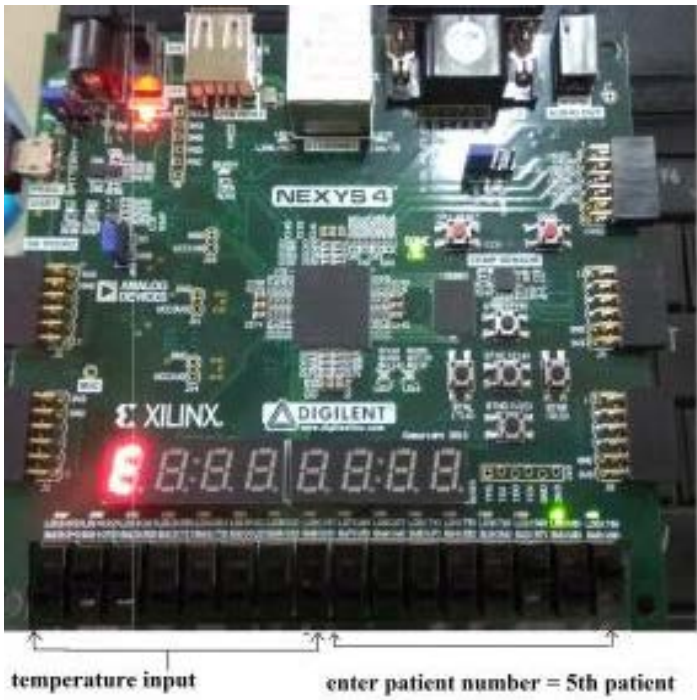

Figure 29. Fifth patient monitoring at emergency condition for temperature.

\section{Acknowledgment}

At the outset I wish to express my gratitude to various people, for their assistance and support, without which it would have been impossible for us to complete our project.

I owe a debt of gratitude towards our mentor Mr. Chetan Chugh who helped and guided me at every step. The project approved by Project approval committee "Runtime Reconfigurability in FPGA for Biomedical Applications." is very interesting and knowledgeable.

I would like to express our thanks to Prof. Bhupinder Verma, Dean of Department of Electronics and Communication Engineering, for allowing us to make use of the laboratories and facilities in it for successful completion of the Dissertation.

I have come to know of many facts about various modules incorporated while making the thesis. Without mentor support and proper guidance this project would have never come into effect and he played a major role in shaping this project.

I would also like to thank my friends who supported 
me while making this project and all their efforts to make this project an effective one. I am also thankful to my parents and Lovely Professional University.

\section{References}

1. Andreasson, Jens et al. Remote system for patient monitoring using Bluetooth ${ }^{\mathrm{Tw}}$. Proceedings of IEEE Vol. 1 Sensors, IEEE; 2002.

2. Arshak, Khalil et al. Modelling remote system for sensor monitoring using Verilog HDL and SIMULINK ${ }^{\circledast}$ co-simulation. Proceedings of the IEEE International BMAS 2005 on Behavioural Modelling and Simulation Workshop. IEEE. 2005.

3. Jones, Phillip $\mathrm{H}$ et al. Dynamically optimizing FPGA applications by monitoring temperature and workloads.20th International Conference on VLSI Design held jointly with 6th International Conference on Embedded Systems. IEEE; 2007.

4. Gama, Óscar et al. Towards a reconfigurable wireless sensor network for biomedical applications. SensorComm 2007 International Conference on Sensor Technologies and Applications. IEEE, 2007.

5. Donald M, Eric J. Runtime FPGA partial reconfiguration. Aerospace Conference, IEEE; 2008.

6. Lie, Wang et al. DPR in FPGAs. Third International Symposium on Intelligent Information Technology Application. 2009; (2).

7. Nasreddine, Nadim et al. Wireless sensors networks emulator implemented on a FPGA. International Conference on Field-Programmable Technology (FPT), IEEE; 2010.

8. Liu, Shaoshan et al. Energy reduction with run-time partial reconfiguration. FPGA; 2010.

9. Mulligan, et al. Coverage in wireless sensor networks: a survey. Network Protocols and Algorithms. 2010; 2(2):27-53.

10. Vipin, Kizheppatt et al. A high speed open source controller for FPGA partial reconfiguration. FPT; 2012.
11. Bhuvaneswari K et al. DPR in low-cost FPGAs. International Journal of Scientific and Engineering Research. 2013 Sep; 4(9):1410-13.

12. Hatai, Indranil et al. FPGA implementation of a fatal heart rate measuring system. International Conference on Advances in Electrical Engineering (ICAEE), IEEE; 2013.

13. Yamaguchi, Shoichi et al. Programmable wireless sensor node featuring low-power FPGA and microcontroller. International Joint Conference on Awareness Science and Technology and Ubi-Media Computing (iCAST-UMEDIA). IEEE; 2013.

14. Meena $S$ et al. Runtime reconfiguration of wireless sensor node using FPGA. International Conference on Computing, Communication and Networking Technologies (ICCCNT). IEEE; 2014.

15. Risman, Nur Sabrina et al. FPGA design and implementation of Electrocardiogram biomedical embedded system. IEEE Conference on Biomedical Engineering and Sciences (IECBES), IEEE; 2014.

16. Meena $S$ et al. Simulation of dynamically reconfigurable wireless sensor node. International Conference on Electronics and Communication Systems (ICECS), IEEE; 2014.

17. Rasu R, Sundaram PS, Santhiyakumari N. FPGA based non-invasive heart rate monitoring system for detecting abnormalities in Fetal. International Conference onSignal Processing and Communication Engineering Systems (SPACES), IEEE; 2015.

18. Rao M, Newe T, Grout I, Lewis E, Mathur A. FPGA based real time 'secure' body temperature monitoring suitable for WBSN.IEEE International Conference on Computer and Information Technology; Ubiquitous Computing and Communications; Dependable, Autonomic and Secure Computing; Pervasive Intelligence and Computing (CIT/ IUCC/DASC/PICOM), Liverpool; 2015. p. 140-3.

19. Borkute CV, Deshmukh AY. Run time DPR using micro blaze soft core processor for DSP applications. International Journal of Research in Engineering and Technology. 2013; 2(12):151-4. 